\title{
Determining the effects of aging on halloysite nano-tube modified binders through the pull-off test method
}

\begin{abstract}
Aging changes the chemistry of bitumen and this adversely affects bitumen's adhesion with aggregate. For this reason, the use of pull-off test was proposed to evaluate bitumen's adhesion during different aging stages. In this study, the pull-off test method is used to evaluate the effects of aging on a 60/70 penetration grade bitumen and binders modified with 2 and 4\% halloysite nano-tube (HNT). The short-term aging (STA) and long-term aging (LTA) of unmodified and modified bitumens were simulated using the rolling thin film oven (RTFO) and pressure aging vessel (PAV) tests respectively. The pull-off test was conducted using the universal testing machine (UTM). Stainless steel and granite substrates moulds were designed and fabricated for this purpose. Dry and wet conditions were simulated to evaluate the loss of bonding strength due to aging. Additionally, consistency tests, namely penetration test, softening point test, and viscosity test, were conducted on all binders. The activation energy (AE) for unaged and aged binders were also evaluated. The results for the consistency test; the pull-off test for stainless steel substrate; and the values for AE showed that the addition of the HNT decreased the effect of aging, which means that modified binders showed decreased temperature susceptibility and this is reflected in improved aging resistance. The result of the pull-off test on granite substrate showed that binders modified with HNT performed better under dry and wet conditions. It can be concluded that HNT is an anti-aging as well as anti-striping agent.
\end{abstract}

Keyword: Bitumen; Nano-clay; Pull-off strength; Activation energy; Aging 